\title{
FLUENT : la modélisation numérique au service de la conception optimisée des réacteurs de post-chloration
}

\section{FLUENT : modelling for optimising the design of post-chlorination tanks}

\author{
par O. Chataigner, J.L. Gagnon \\ Générale des Eaux \\ G. Chagneau, P. Chopard \\ Syndicat des Eaux d'Ile-de-France
}

The existence of increasingly powerful computers at an affordable price has led to the extended use of Computational Fluid Dynamics (CFD), initially developed for the aeronautics sector. The study presented here illustrates the use of a calculation code that is readily available in the retail sector. FLUENT is used to evaluate the hydraulic efficiency of chlorination tanks. The aim is to provide a homogeneous flow within the tank, a measure needed to ensure good water treatment quality. The simulations shown demonstrate that the use of a CFD code is an actual engineering tool and has led to practical recommandations for the construction of chlorination tanks.

\section{I — SITUATION DE L'ÉTUDE ET OBJECTIFS}

Les usines de production du Syndicat des Eaux d'Ile-deFrance utilisent les eaux de surface de l'Oise, de la Marne et de la Seine qui nécessitent un traitement complexe avant de fournir un produit conforme aux normes de potabilité. La chloration, dernière étape de cette chaîne de traitement avant l'introduction de l'eau dans le réseau, assure la désinfection finale de l'eau et la satisfaction d'une partie de la demande en chlore de l'eau. On distingue usuellement deux catégories de réservoirs :

- Les réacteurs de post-chloration sont les unités où se produit le contact entre le chlore et l'eau. Ils fonctionnent en régime permanent. Leur rôle est d'assurer une désinfection satisfaisante, tout en limitant la formation de sous-produits de chloration.

- Les réservoirs d'effacement permettent de prolonger les réactions précédentes et de stocker l'eau avant la distribution dans le réseau. Ils fonctionnent en régime variable.

La circulation de l'eau au sein des réservoirs de chloration conditionne la qualité de la désinfection et influe directement sur le temps de contact eau-chlore. En fonction de la géométrie de ces ouvrages et de leur sollicitation hydraulique, la dispersion des temps de séjour sera plus ou moins importante et aura une grande influence sur la qualité du traitement.

Deux approches complémentaires permettent de déterminer les distributions de temps de séjour. Le traçage, méthode de terrain, permet un diagnostic efficace des installations existantes. Cette technique n'est cependant pas utilisable pour déterminer les performances des ouvrages en projet. La seconde approche consiste à utiliser un code de résolution des équations de la mécanique des fluides pour simuler l'hydraulique de ces ouvrages de contact. Cette technique permet également d'envisager des situations variées en termes de débits, hauteurs d'eau et aménagements intérieurs.

L'étude présentée ici a pour objectifs :

- d'expliquer les principes et moyens à mettre en œuvre pour simuler numériquement le comportement hydraulique des réservoirs de chloration ;

- d'exposer le gain hydraulique procuré par des aménagements intérieurs aux réservoirs de chloration.

\section{II — SITES ÉTUDIÉS}

\subsection{Réservoir EF de Méry}

Le projet “ Méry 340000 " prévoit l'implantation d'un nouveau réservoir de post-chloration appelé EF d'une capacité de $10500 \mathrm{~m}^{3}$, qui assurera la désinfection finale des eaux issues de la filière biologique sur charbon actif en grains. Le fonctionnement de cette étape de désinfection doit permettre de garantir le respect des normes microbiologiques (germes tests de contamination fécale) et chimiques (sousproduits de chloration).

D'un point de vue hydraulique, l'objectif est d'avoir un réservoir dans lequel l'eau évolue de façon uniforme de l'entrée vers la sortie, en limitant les phénomènes de recirculation. Ces phénomènes induisent, en effet, une dispersion des temps de séjour préjudiciable à une bonne maîtrise de la qualité des eaux produites. Les différents aménagements 


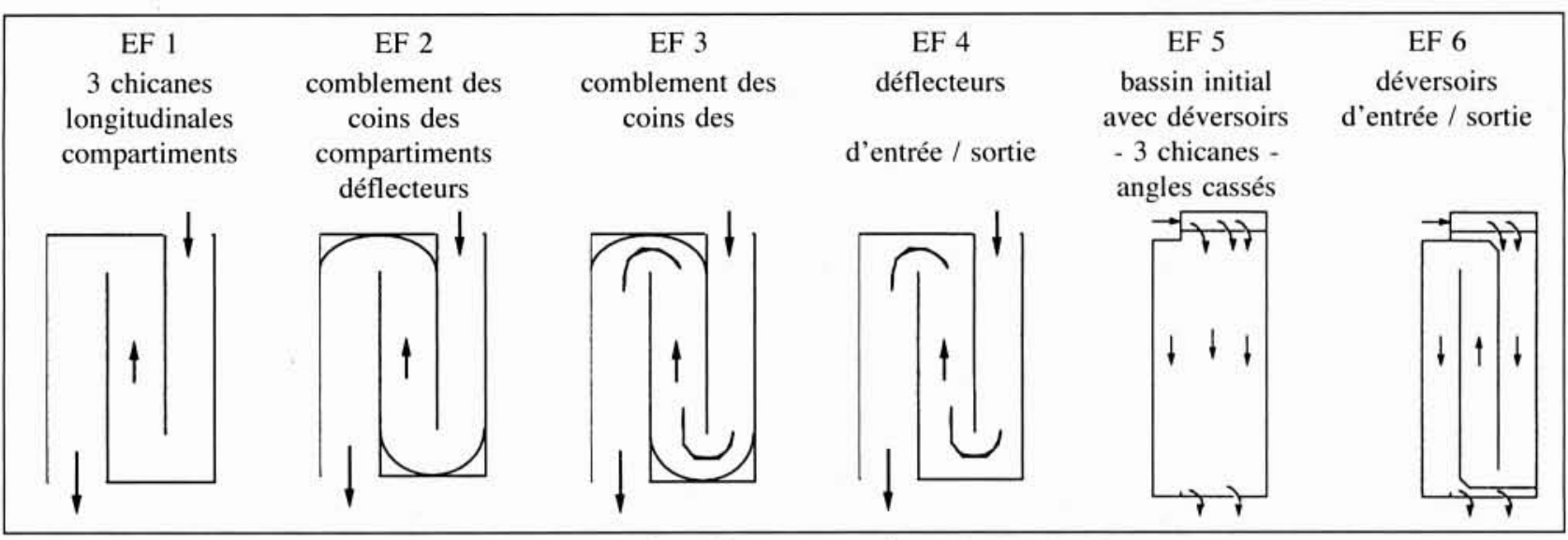

1. Configurations étudiées pour le réservoir EF de Mery.

évalués pour atteindre cet objectif combinent l'implantation de chicanes longitudinales avec des déflecteurs et/ou un comblement des zones mortes dans les angles des chicanes. Ils sont représentés sur la Figure 1. Suite à une première évaluation de ces différents aménagements, une étude plus détaillée a été réalisée intégrant notamment les déversoirs d'entrée et de sortie.

Les configurations de réservoir ont été simulées au débit minimal de $30000 \mathrm{~m}^{3} / \mathrm{j}$, au débit nominal de $100000 \mathrm{~m}^{3} / \mathrm{j}$ et au débit maximal de $200000 \mathrm{~m}^{3} / \mathrm{j}$.

\subsection{Réservoir D de Choisy}

La création du réservoir d'effacement $\mathrm{D}$ d'un volume de $35000 \mathrm{~m}^{3}$ à l'usine de Choisy-le-Roi vise à améliorer la désinfection, l'effacement et la mise à l'équilibre de l'eau. De même que pour le réservoir EF de Mery, on recherche les aménagements permettant d'obtenir un écoulement homogène entre l'entrée et la sortie du réservoir. A cette fin, deux configurations de chicanage ont été étudiées ; elles sont représentées sur la Figure 2. La configuration D 2 a un ratio d'élancement (longueur / largeur d'un compartiment) supérieur à celui de l'aménagement D 1 .

Ces deux configurations ont été simulées au débit nominal de $400000 \mathrm{~m}^{3} / \mathrm{j}$ et/ou au débit maximal de $800000 \mathrm{~m}^{3} / \mathrm{j}$ pour la hauteur d'eau minimale $(1,8 \mathrm{~m})$ et/ou maximale $(5 \mathrm{~m})$.

\section{III — MATÉRIEL ET MÉTHODES}

\subsection{Notion de distribution des temps de séjour}

L'eau séjourne dans le réservoir, pendant un temps de séjour théorique $t$ égal au volume du réservoir divisé par le débit entrant. Cependant, les masses d'eau qui traversent le réservoir suivent des parcours différents. Certaines de ces masses d'eau vont suivre des parcours préférentiels dans ce réservoir et sortir du réservoir avant le temps $t$ tandis que d'autres vont suivre des zones de recirculation et sortir du réservoir après le temps $t$. Ainsi, un réacteur peut être caractérisé par la distribution des temps de passage des différentes masses d'eau entrées simultanément dans le réservoir.

Cette Distribution des Temps de Séjour (DTS) peut être déterminée par un suivi des différentes masses d'eau transitant dans les ouvrages et est caractérisée par les paramètres temporels suivants [1-5] :

$\tau \quad: \quad$ temps de séjour théorique (Volume / Débit)

$T 10$ : temps de sortie de $10 \%$ du volume entrant

T90 : temps de sortie de $90 \%$ du volume entrant

$\mathrm{Tg} \quad$ : temps de passage moyen

d : indice de dispersion

L'écart à l'écoulement piston peut être caractérisé par le rapport $T 10 / \mathrm{Tg}$. Ce rapport est privilégié, car il est utilisé dans le calcul des $C^{*} T$ (résiduel de chlore* temps de contact) défini selon la législation américaine par l'USEPA (United States Environmental Protection Agency) [6]. Plus ce rapport est proche de 1, plus l'écoulement est piston. La suite des études hydrauliques a donc été menée de façon à comparer les configurations géométriques retenues à l'aide du rapport $T 10 / T g$.

Le ratio $T 90 / T g$ est caractéristique du taux de recirculation du réservoir. Il indique le temps de transit de $90 \%$ du fluide par comparaison au temps de séjour moyen. Cet indice apporte donc une information supplémentaire pour quantifier l'écart à l'écoulement piston. L'indice T90/T10, appelé indice de Morril, est un indicateur du degré de mélange du réservoir.

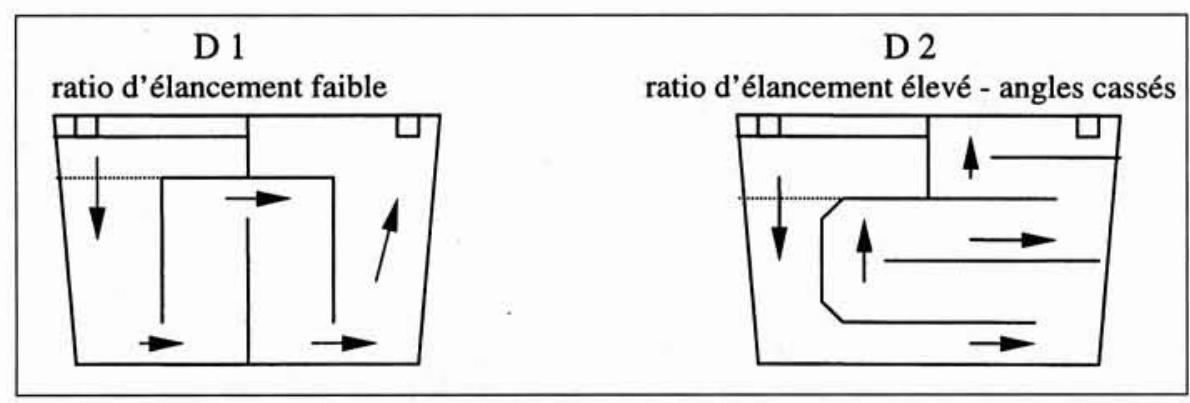

2. Configurations étudiées pour le réservoir D de Choisy. 


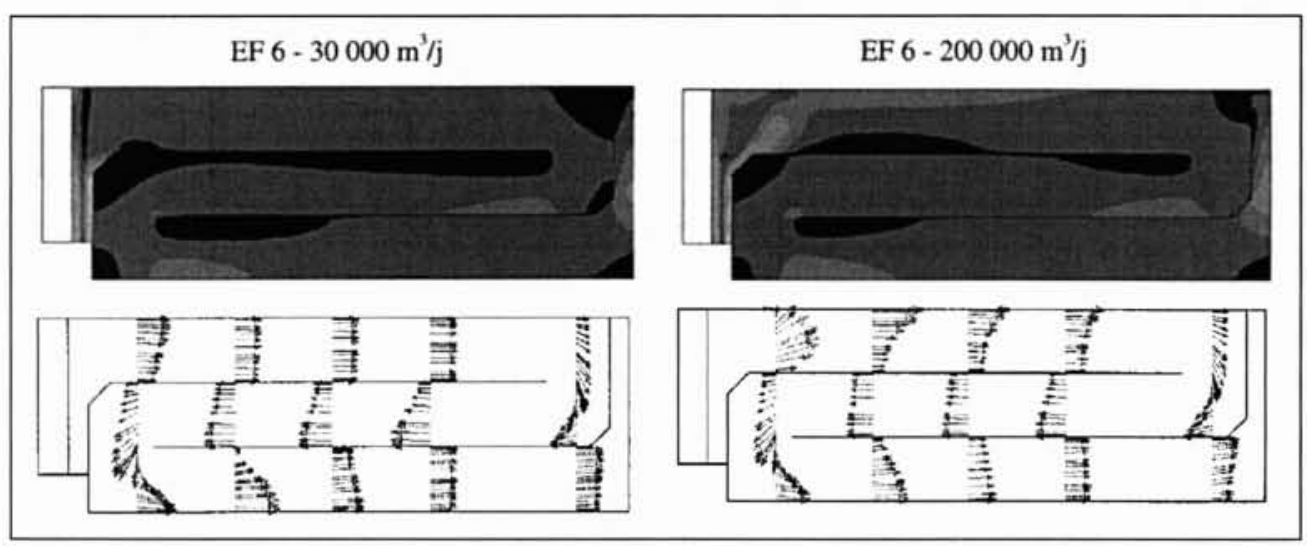

3. Visualisation des écoulements pour la configuration MERY 6 - courbes d'iso-vitesse et vecteurs vitesse.
Enfin, l'indice de dispersion, lié à la variance de la courbe de distribution des temps de séjour, est un indicateur complémentaire d'écart au flux piston pour lequel $d$ tend vers 0 .

\subsection{Logiciel FLUENT et équipement informatique}

Les simulations numériques ont été réalisées sur une station de travail HP735-125 (144 Mo de RAM, $125 \mathrm{MHz}$ ) avec une licence d'utilisation de Fluent (Geomesh version 3.0 et Uns version 4.1). Le logiciel Fluent est un code généraliste de résolution des équations de la mécanique des Fluides. Il repose sur la résolution par une méthode de volumes finis des équations de Navier-Stokes, complétées par un modèle de turbulence à deux variables $(k-\varepsilon)$ et une équation d'advection-diffusion d'espèce [7].

\section{- 3.3 Détermination du comportement hydrodyna- mique des réservoirs}

On distingue usuellement trois étapes dans la simulation numérique des écoulements au sein des réservoirs.

Le prétraitement consiste à définir le problème que l'on souhaite traiter. Les grandes étapes sont la définition de la géométrie du réservoir que l'on souhaite étudier et la génération du maillage associé. L'étape de maillage représente la partie la plus importante du travail puisque la qualité des résultats obtenus en dépend directement. Les numériciens considèrent usuellement que cette étape nécessite $70 \%$ du temps de travail total. Le volume occupé par l'eau est tout d'abord compartimenté en un ensemble de blocs sur lesquels des nœuds sont ensuite répartis. On obtient alors une subdivision du réservoir permettant d'appliquer la résolution par volumes finis. Au cours de cette étape de mise au point du maillage, on cherche à obtenir une répartition de nœuds la plus homogène possible et à éviter des distorsions trop importantes dans la forme des mailles. Des critères tels que l'inclinaison des mailles et le rapport des tailles des cellules adjacentes sont des aides utiles pour évaluer la qualité du maillage mis au point.

Le solveur permet de caractériser les différents éléments physiques du réservoir à modéliser et de calculer l'écoulement hydraulique associé. C'est à cette étape que l'on définit les conditions aux limites du modèle du réservoir et que l'on ajuste les paramètres de turbulence. Au cours de cette étude, le débit a été imposé en définissant une vitesse constante sur la section d'entrée. La condition de sortie est une pression constante. La surface libre a été assimilée à une zone de symétrie, afin d'annuler les vitesses normales.

Deux types de conditions de fonctionnement peuvent être choisies :
- les conditions les plus défavorables pour l'hydraulique sont considérées pour effectuer un calcul pessimiste et vérifier le fonctionnement dans la situation la plus critique ;

- les conditions de fonctionnement nominales pour connaitre la situation la plus généralement obtenue dans le réservoir.

Après la résolution des équations de la mécanique des fluides contrôlée par le suivi de critères de convergence, on obtient le champ de vitesse à l'intérieur du réservoir.

Le post-traitement permet ensuite d'obtenir une visualisation d'ensemble de l'écoulement du fluide au sein du réservoir simulé. On caractérise ainsi les zones de court-circuit et de recirculation préjudiciables à une bonne maîtrise de la qualité des eaux produites.

\subsection{Obtention des distributions de temps de séjour avec FLUENT}

Un calcul complémentaire permet d'accéder aux caractéristiques temporelles. On utilise pour cela les écoulements préalablement établis pour simuler la propagation d'un traceur dans le réservoir.

L'opération se fait en deux temps : on réalise d'abord une injection d'une deuxième fraction d'eau, repérée par un nom différent, pendant une durée courte. La fraction massique de ce flux injecté est définie comme condition aux limites au niveau de l'entrée. Cette deuxième fraction d'eau représente le traceur et possède les mêmes caractéristiques que l'eau du réservoir. La concentration de cette deuxième fraction de fluide est ensuite calculée dans le flux de sortie au cours du temps.

Les données de concentration au cours du temps sont alors traitées automatiquement à l'aide d'une macro mise au point sous Excel pour obtenir directement les différents paramètres temporels précédemment définis.

\section{RÉSULTATS}

\section{- 4.1 Etude hydrodynamique des réservoirs}

La visualisation des champs de vitesse nécessite la création de surfaces de projection pour analyser les problèmes en trois dimensions. Des surfaces horizontales et transversales ont été utilisées pour analyser les champs de vitesse. Les courbes d'iso-vitesse et les graphes de vecteurs vitesse sont représentés sur les figures 3 et 4 .

\subsection{Paramètres temporels}

L'analyse de l'efficacité hydraulique consiste à déterminer la distribution de temps de séjour donnée par le traçage. Les figures 5 à 8 récapitulent les valeurs des paramètres temporels pour les différents débits et/ou hauteurs d'eau. 


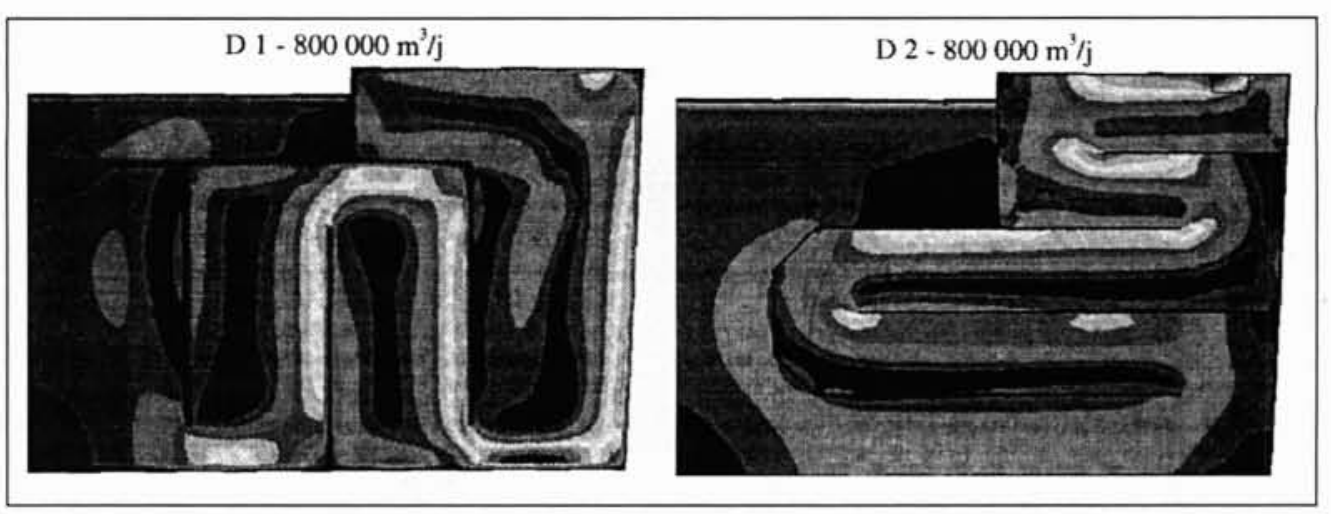

4. Visualisation des écoulements pour les configurations de Choisy - courbes d'isovitesse.

\section{$\mathrm{V} \square$ DISCUSSION}

\section{- 5.1 Comparaison des aménagements intérieurs}

La première phase d'étude pour le réservoir EF de Méry a pour but de comparer différents aménagements à base de chicanes longitudinales complétés par des déflecteurs et/ou un comblement des zones mortes dans les angles des chicanes. Ces simulations ne prennent pas en compte les déversoirs d'entrée et de sortie. Il ressort que l'utilisation de chicanes longitudinales est un moyen efficace et satisfaisant pour obtenir un comportement hydraulique proche du flux piston.

Les configurations testées (EF 1 à 4) permettent d'obtenir un écoulement proche du flux piston. Le ratio d'efficacité hydraulique $T 10 / T g$, caractéristique du taux de court-circuit au sein du réservoir, est en effet supérieur à 0,8 pour la plupart des débits simulés. Cet indice est égal à 1 pour un flux idéalement piston. Dans les cas étudiés, cela signifie que $10 \%$ de l'eau transite dans le réservoir en un temps inférieur à 0,8 fois le temps de séjour moyen. L'indice de Morril T90/T10, indicateur du degré de mélange au sein du réservoir, est en général inférieur à 1,6 pour ces configurations. Cela illustre la faible dispersion des temps de séjour rendue possible par ces aménagements testés. Le type de comportement obtenu est donc tout à fait satisfaisant par rapport aux objectifs visés.

Le comblement des angles (EF 2 \& 3) n'apporte pas de gain suffisant pour justifier l'implantation de tels dispositifs. La réalisation de cet aménagement est en effet délicate et elle entraîne de plus une diminution du volume du réservoir et donc des temps de contact. Les simulations montrent que le gain hydraulique procuré par cet aménagement ne compense pas cette diminution.

De la même manière, l'ajout de déflecteurs (EF 3 \& 4) dans les virages en $U$ n'est pas nécessaire pour assurer une bonne maîtrise des temps de contact. Ces installations ne sont réellement justifiées que pour le cas où le débit est maximal $\left(200000 \mathrm{~m}^{3} / \mathrm{j}\right)$. Le facteur d'élancement (longueur/largeur) de chaque compartiment est donc suffisant pour retrouver rapidement un écoulement homogène.

Il ressort donc que des performances hydrauliques satisfaisantes sont obtenues grâce à l'utilisation de chicanes longitudinales (EF 1).

\subsection{Influence du chicanage sur l'efficacité hydraulique}

Sur la base de ces résultats, différents chicanages ont été étudiés afin de parvenir à un design optimal de ces aménagements. Les paramètres à optimiser pour améliorer le comportement hydraulique sont d'une part le ratio d'élancement, d'autre part la limitation des angles susceptibles de perturber l'écoulement.
L'étude du réservoir EF de Méry (EF 6) montre que l'implantation de 3 chicanes améliore de façon significative l'efficacité hydraulique du réservoir. Les angles droits du compartiment central ont été éliminés afin de faciliter I'homogénéité des écoulements de part et d'autre de la chicane (cf. Figure 3). Cette configuration a été testée en prenant en compte les déversoirs d'entrée et de sortie et a été comparée à un réservoir sans aménagement particulier. Au débit de $200000 \mathrm{~m}^{3} / \mathrm{j}$, l'efficacité hydraulique est doublée avec un ratio $T 10 / T g$ qui passe ainsi d'une valeur initiale (EF 5) de 0,35 à une valeur de 0,68 . Les courts-circuits sont donc considérablement réduits avec ce type d'aménagement. De manière similaire, I'utilisation de chicanes limite l'importance des recirculations : le ratio $T 90 / T g$ passe d'une valeur de 1,95 à une valeur de 1,33. Cela signifie que $90 \%$ du flux transite dans le réservoir en 1,33 fois le temps de séjour moyen à comparer à la valeur initiale de 1,95 fois le temps moyen. L'aménagement de chicane permet donc une faible dispersion des temps de séjour autour du temps de séjour moyen.

L'étude réalisée sur le réservoir D de Choisy-le-Roi permet de confirmer le bien-fondé de ce type d'aménagement. Une première configuration de chicanage (D 1) a été testée pour répartir le flux dans le réservoir. De même que précédemment, les phénomènes de court-circuit préjudiciables d'un point de vue bactériologique sont diminués par cet aménagement. Cependant l'étude hydraulique montre que le chicanage transversal, caractérisé par un rapport d'élancement (longueur / largeur de compartiment) faible, favorise les zones de recirculation dans la partie située à l'arrière de chaque chicane. Pour restreindre ce phénomène de recirculation, une seconde configuration (D 2) possédant un ratio d'élancement supérieur a été étudiée. Les angles liés aux chicanes ont été cassés pour le compartiment d'entrée pour lequel l'opération pouvait être réalisée sans difficulté. Les courbes d'iso-vitesse (cf. Figure 4) montrent la diminution des zones de recirculation. L'efficacité hydraulique se trouve ainsi améliorée d'un facteur 1,5 avec un ratio $T 10 / T g$ augmenté de 0,2 pour atteindre une valeur proche de 0,6 . L'écoulement est donc plus homogène dans cette seconde configuration.

\subsection{Influence du débit}

L'influence du débit sur l'efficacité hydraulique a été testée pour les réservoirs chicanés. Un changement du régime hydraulique modifie en effet les écoulements intérieurs aux réservoirs et pourrait engendrer une dégradation des performances obtenues. Les résultats montrent l'homogénéité des paramètres temporels sur toute la gamme de débits envisagée. Les temps de séjours sont ainsi peu dispersés grâce à un aménagement de chicanes et leurs valeurs sont proportionnelles à l'inverse du débit. 


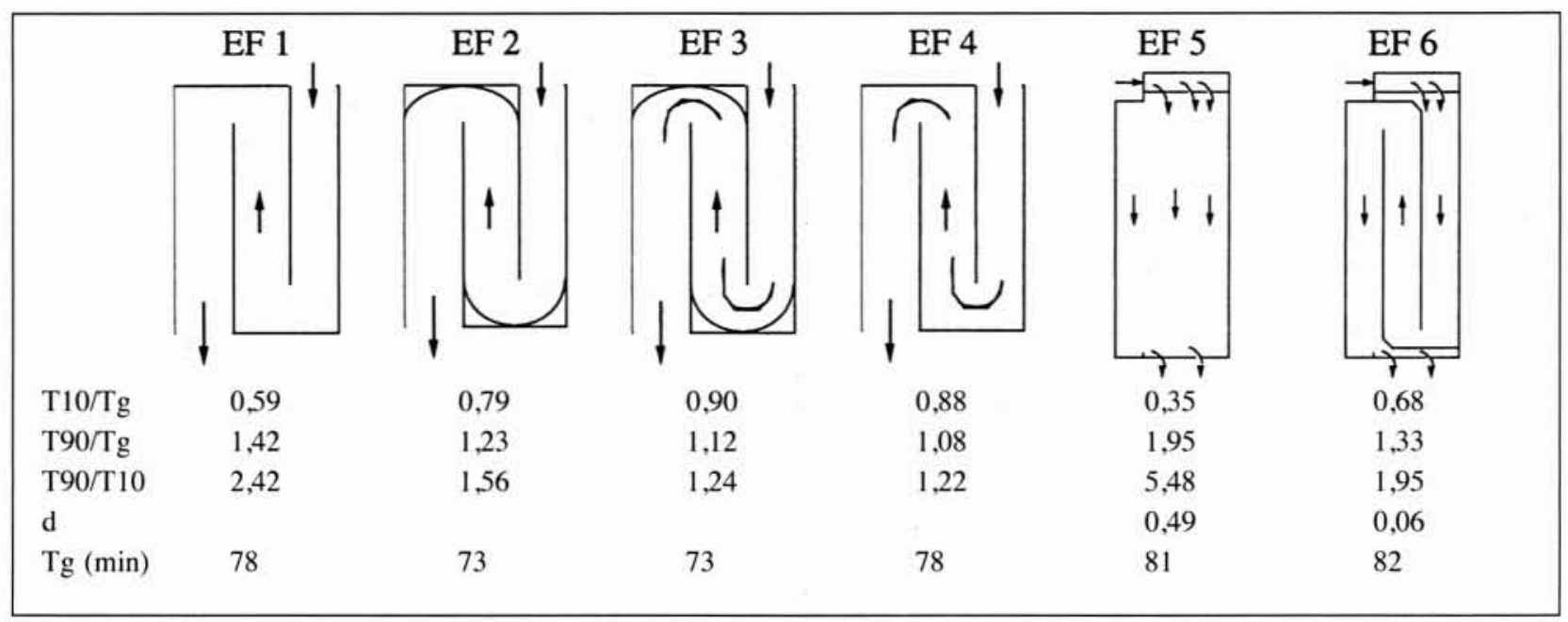

5. Paramètres temporels - réservoir EF de Mery - $Q=200000 \mathrm{~m}^{3} / \mathrm{j}$.

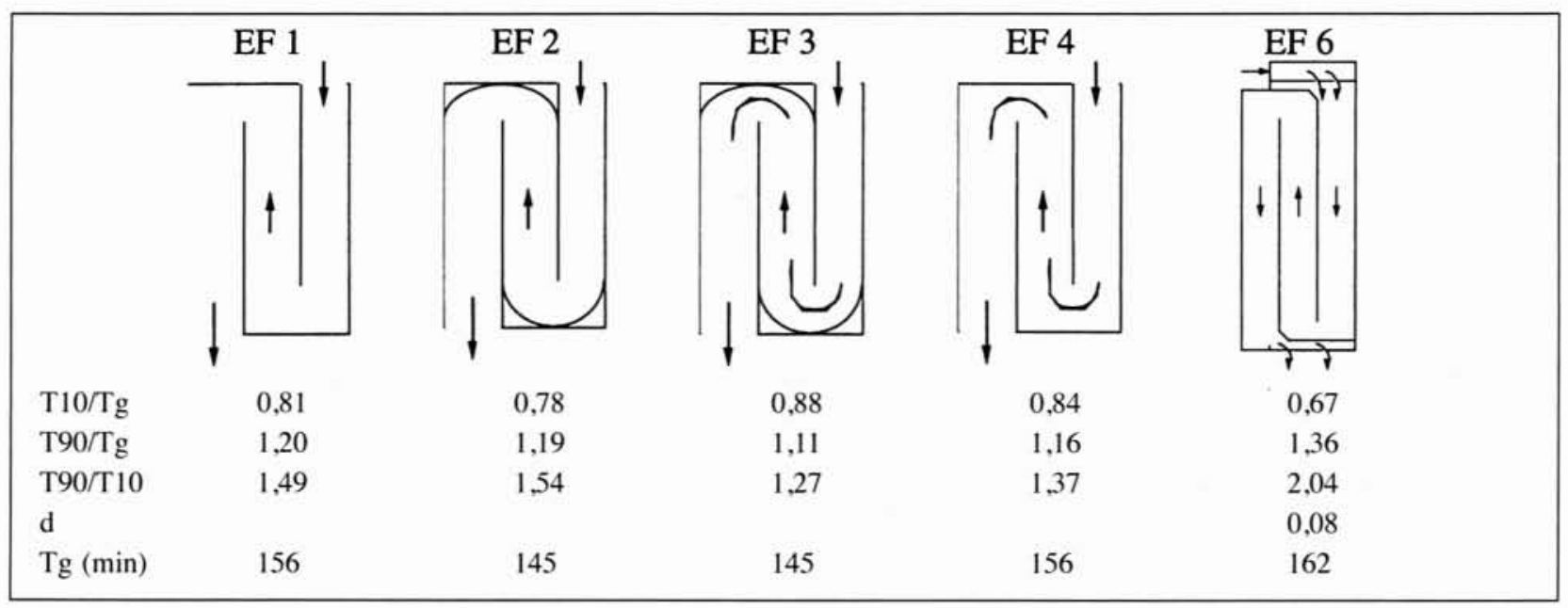

6. Paramètres temporels - réservoir EF de Mery - $Q=100000 \mathrm{~m}^{3 / 3}$.

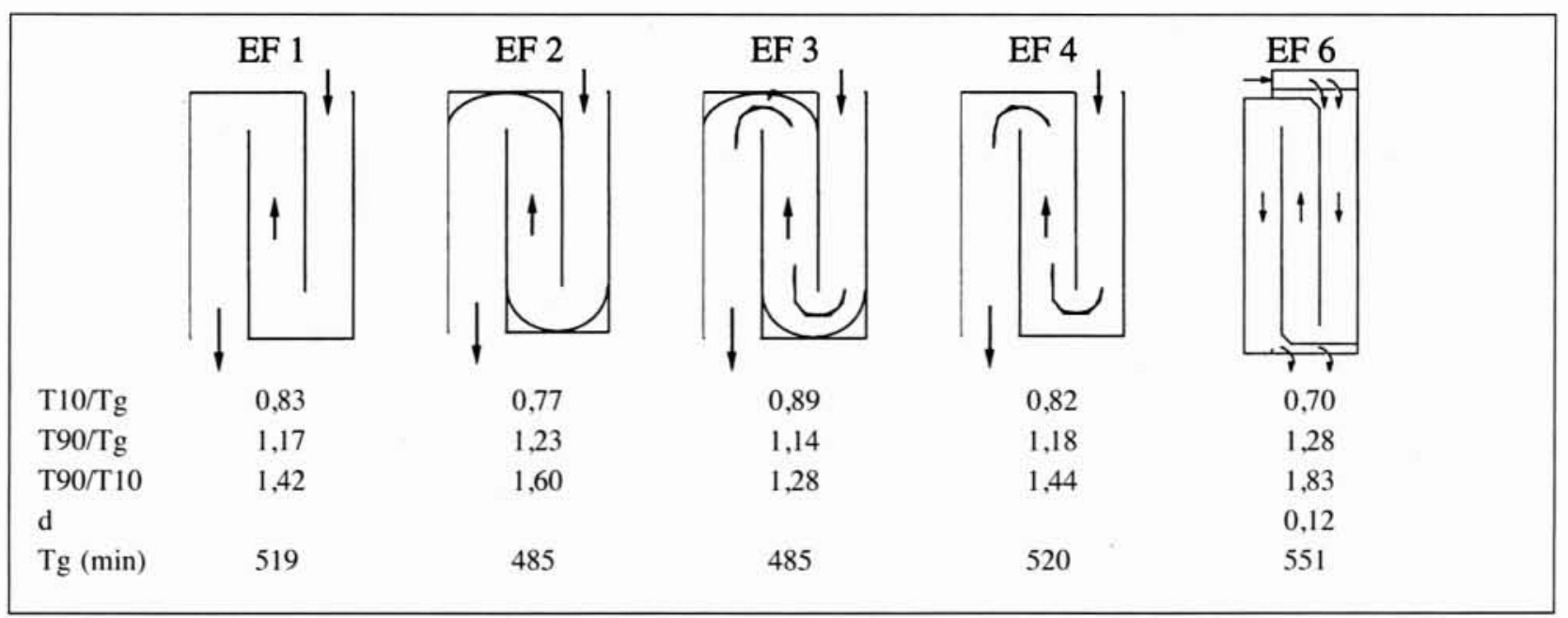

7. Paramètres temporels - réservoir EF de Mery - $Q=30000 \mathrm{~m} 3 / \mathrm{j}$. 


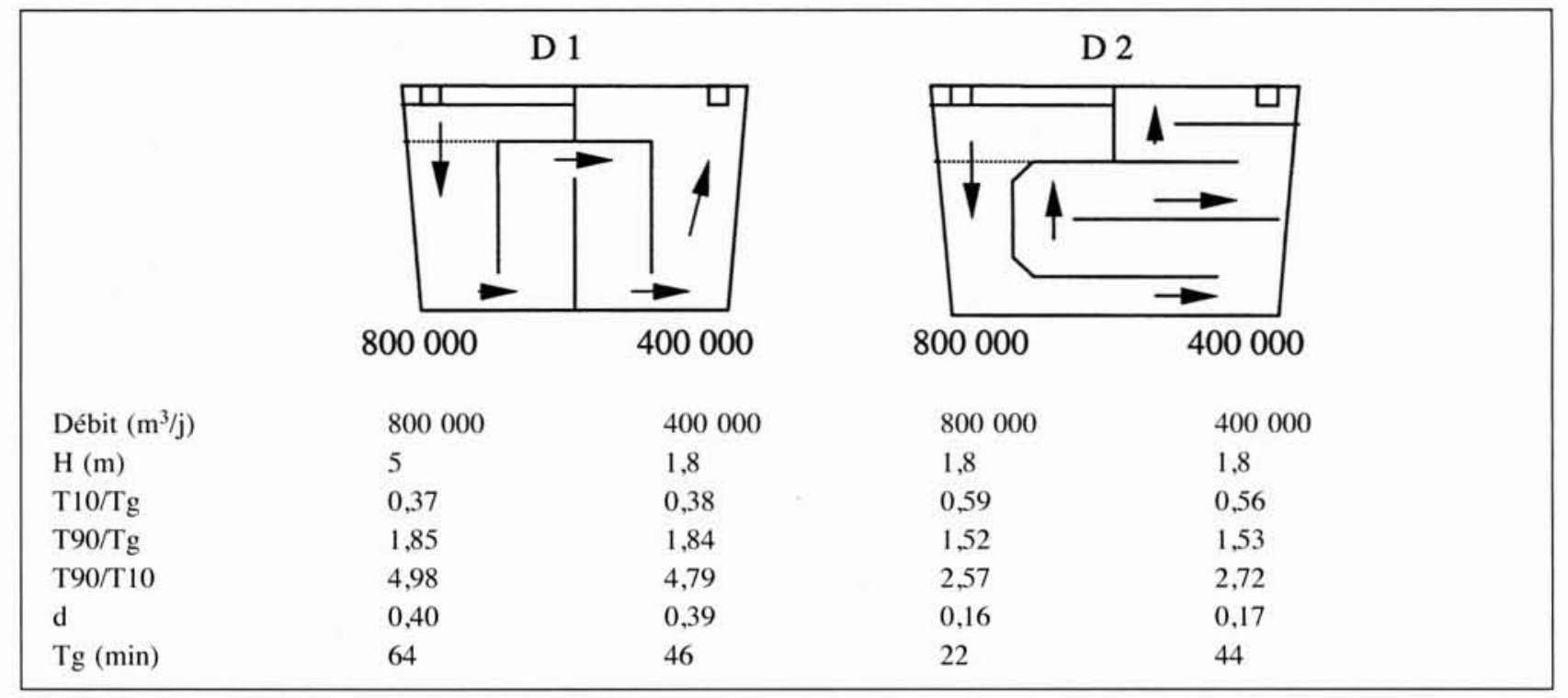

8. paramètres temporels - réservoir D de Choisy.

Les performances hydrauliques obtenues sur le réservoir EF de Méry-sur-Oise (EF 6) restent constantes d'un débit de $30000 \mathrm{~m}^{3} / \mathrm{j}$ à un débit de $200000 \mathrm{~m}^{3} / \mathrm{j}$. Le ratio $T 10 / T g$ est ainsi très peu affecté par les modifications de régime et sa valeur reste comprise entre 0,67 et 0,70 d'une simulation à l'autre. On observe par contre une augmentation des recirculations pour le débit minimal. Cette augmentation des recirculations pour les débits faibles est caractérisée par l'augmentation de l'indice de dispersion qui est doublé entre le débit minimal et le débit maximal pour atteindre une valeur de 0,12 . Les valeurs obtenues restent cependant satisfaisantes puisque le taux de court-circuit est peu affecté par cette modification de régime.

La même tendance est observée sur le réservoir D de Choisy-le-Roi. La configuration retenue (D 2) a en effet été évaluée dans la situation critique où la hauteur d'eau est la plus faible possible. On observe des performances similaires au débit nominal de $400000 \mathrm{~m}^{3} / \mathrm{j}$ et au débit maximal de $800000 \mathrm{~m}^{3} / \mathrm{j}$. L'aménagement de chicanes longitudinales permet ainsi une bonne maîtrise des temps de contact sur l'ensemble de la plage de débit.

\section{VI $\square$ CONCLUSIONS}

L'étude a permis d'illustrer l'utilisation de moyens numériques pour dimensionner de nouveaux réservoirs de chloration. En ce qui concerne l'efficacité des aménagements testés, on confirme que l'implantation de chicanes longitudinales au sein des réservoirs de chloration est un moyen efficace pour restreindre la dispersion des temps de séjour. Il est souhaitable de supprimer les angles droits des compartiments situés à l'intérieur du réservoir afin de favoriser l'homogénéité des écoulements de part et d'autre des chicanes. Par contre l'ajout complémentaire de déflecteurs dans les virages en U n'est pas nécessaire pour les cas envisagés où le ratio d'élancement (longueur sur largeur d'un compartiment) est élevé. Le comblement des coins des compartiments n'est également pas justifié car il entraîne une diminution du volume utile du réservoir.

Les codes de calcul de mécanique des fluides sont une aide appréciable pour faciliter l'étude des bassins de chloration et pour visualiser l'effet des aménagements envisagés.
Ils permettent d'obtenir une représentation des phénomènes hydrauliques sous réserve d'un travail approprié du maillage. La fiabilité des résultats est en effet corrélée à la qualité de celui-ci, et il peut être nécessaire de rectifier des cellules engendrant un résultat non satisfaisant. Les critères de qualité de maillage sont utiles pour mener à bien ces missions d'expertise et pour identifier les zones sensibles qui doivent être travaillées ou reconfigurées. Ainsi, les logiciels d'analyse numérique sont des outils d'aide à la décision précieux aux professionnels de la mécanique des fluides. Paradoxalement, l'amélioration des codes de calculs et des performances informatiques influe peu sur le temps d'expertise nécessaire à ce type de projet en raison de la meilleure précision visée. La hauteur des investissements réalisés et leurs longévités justifient cette recherche de fiabilité et de finesse dans les résultats obtenus. L'amélioration de la qualité de l'eau distribuée est à ce prix. Concrètement, on attend de ces investissements une diminution des sous-produits de chloration et une meilleure désinfection, notamment lorsque les ressources en eaux brutes se dégradent.

\section{BIBLIOGRAPHIE}

[1] M. M. Bishop, J. M. Morgan, B. Cornwell, D. K. Jamison, Improving the disinfection detention time of a water plant clearwell, JAWWA, vol 85 . $\mathrm{n}^{\circ}$ 3, pp 68-75 (Mars 1993)

[2] P. F. Boulos, W. M. Grayman, R. W. Bowcock, J. W. Clapp, L. A. Rossman, R. M. Clark, R. A. Deininger, A. K. Dhingra, Hydraulic mixing and free chlorine residual in reservoirs, JAWWA, vol 88, $n^{\circ} 7, \mathrm{pp} 48-59$ (Juillet 1996)

[3] F. L. Hart, R. Allen, J. DiAlesio, J. Dzialo, Modifications improve chlorine contact chamber performance Part 1 \& Part 2, Water \& Sewage Works, vol 122, $\mathrm{n}^{\circ} 9$, pp 73-75 (Septembre 1975) \& vol 122, $\mathrm{n}^{\circ} 10$, pp 88 90 (Octobre 1975)

[4] V. Kothandaraman, R. L. Evans, Hydraulic model studies of chlorine contact tanks, Journal of the Water Pollution Control Federation, vol 44 $\mathrm{n}^{\circ} 4$, pp 625-633 (Avril 1972)

[5] D. M. Markse, J. D. Boyle, Chlorine contact chamber design - A field evaluation, Water \& Sewage Works, vol 120, $\mathrm{n}^{\circ} 1$, pp $70-77$ (Janvier 1973)

[6] United States Environmental Protection Agency, Guidance Manual for compliance with the filtration and disinfection requirements for public water systems using surface water sources (Mars1991)

[7] User's Guide for FLUENT/UNS \& RAMPANT 